\title{
Construction d'un remblai routier expérimental en résidus de bauxite
}

\section{A. MOURATIDIS}

Université Aristote de Thessalonique 54124 Thessalonique. Grèce tasosiohermes.civil.auth.gr

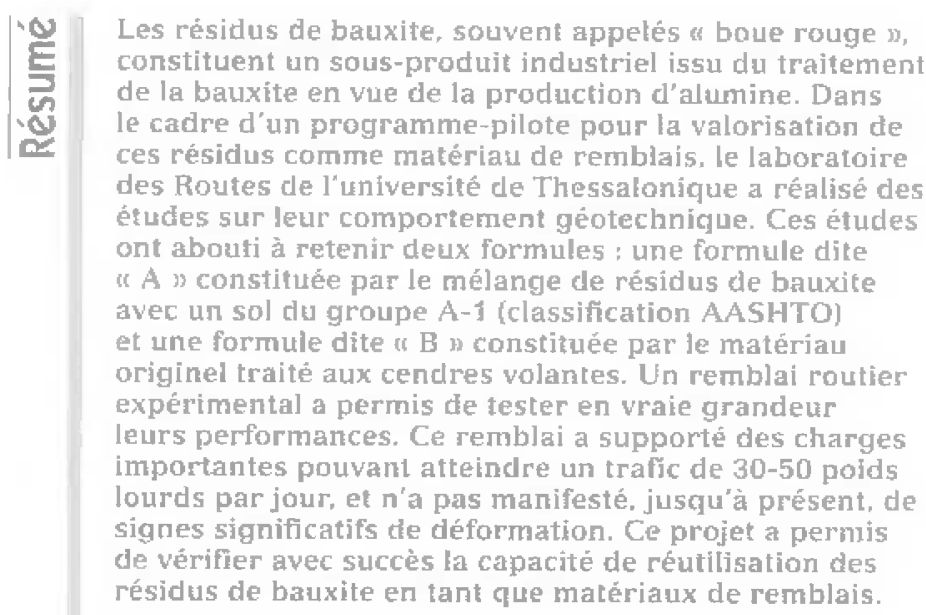

Mots-clés : résidus de bauxite, remblai, construction, route, matériau, trafic, terrassements.

\section{Construction of an experimental highway embankment by bauxite residues}

Bauxite residues, often called "red mud". constirute a by-product of bauxite processing for the producton of alumina. Within the frame of an experimutal research for the utilization of bauxite residues as a road embankment material. the Highway Engineering Laboratory of the University of Thessaloniki has performed studies on their geotechnital performance. These studies led to two potential formulas to apply on site: the flrst one, called "A" consists of a mixture of bauxite residues and a soil A-1 [classification A.SHTO), the second comprises the material in its original condition treated by fly-ash. This earth structure, subjected to heavy loading. corresponding to 30-50 trucks daily, has not exthibited any signs of distress or deformation. The pilot-project, meant to provide evidence of potential use of bauxite residues as a road embankment material, was proven by its performance assessment to be successful and promising.

Key words: bauxite reşidues, embankment. construction, road, material, traffic, earthwork. 


\section{Introduction}

Les résidus de bauxite, souvent appelés a boue rouge n, sont générés suivant le procédé industriel du traitement de la bauxite en vue de la production de l'alumine. En Boétie, l'usine d'a Aluminium de Grèce " produit environ 700 000 tonnes de résidus de bauxite par an, donl une grande partie est amenée par pompage et dispersée, sous forme liquide, en mer. En effet, tous les pays gros producteurs de lésidus de bauxite [Etats- ¿nis, Australie, Japon, Indel suivent la praticue de a disposition " ou de urejet p plutót que celle du recyclage malgré la sensibilisation générale pour les problemes d"environnement.

Que ce soit dans la métallurgie ou dans l'industrie des matériaux de construction, la quantité totale recyclée reste juscu'à présent trés limitée, voire infíme Cette constatation s'expligue par le coût du transport du sous-produit juscu'à un site de recyclage ou de réutilisation et aux difficultés pratiques que pose son utilisation. Il est également vail que le comportement de ce matériau demeure de nos jours mal connu.

Les principaux domaines de valorisation des résidus de bauxite sont actuellement la production de ciments colorés, la fabrication de briques et de tuiles, le confinement de déchets toxiques. l'extraction d'éléments minéraux. Les applications en construction routière occupent une place secondaire; les cas réels d'application du sous-produit à un projet routier sont rares. Un premier projet-pilote d'utilisation de résidus de bauxite en technique routiêre a été réalisé par le laboratoire régional des Ponts et Chaussées d'Aix-en-Provence a Вramefan (1995). II s'agissait d'une construction de remblai en résidus de bauxite, stabilisés par cendre volante, d'une longueun de $300 \mathrm{~m}$ et d'une hauteur de 1 n (LRPC d"Aix-en-Provence, 1997).

La vajorisation dans je domaine des travaux routiers semble prometteuse. car cette activité présente l'avantage de pouvoir absorber cle grandes quantités de matériaux, comparée aux autres domaines de valorisation (Jiłsangiam et al., 2008). Étant donné que l'objectif des operations de recyclage est d"introduine des produits secondaires aux ouvrages technigues à un taux de volume de réutilisation élevé, la construction routière constitue ainsi un enjeu d'application majeur. Poulv favoriser l'utilisation de ces residus, des recher' ches en laboratoire et en conditions de chantier sont nécessaires.

\section{Recherches en laboratoire}

A l'université de Thessalonique, le laboratoire des Routes a entrepris un premier projet de recherche sur les résidus de bauxite en 1993. Ce projet consistait à déterminer lés propriétés du sous-produit industriel en laboratoire ainsi que ses effets de stabilisation sur différents types de sol dans la perspective d'une réutilisation dans differents domaines de la construction d'ouvages en terre : remblais, barrages, barrières étanches de confinement de déchets toxiques.

Les résidus de bauxite de l'usine d'« Aluminium de Grèce ju sont sinilaires, de par leur composition chimique, à ceur des Etats-Unis, contenant un pourcentage élevé d'oxyde ferrique [Tableau I]. Des essais d'alcalinité ont donné une valeur moyenne de $\mathrm{pH}=11$.

Quant aux propriétés physiques du matêriau, lanalyse en taboratoire a fourni des caractéristigues d"un silt non plastique. Les valeurs de la limite de liquidité variaient de 35 a 39 . Sur un nombré limité d'essais. ta limite de plasticité a pu être déterminée, $w_{+}=33-35$, ce qui conduirait à un indice de plasticité $L=4$. Pourtant, sur la majorité des essais, la limite de plasticité n'a pu être déterminée et le matériau a êté considêré comme non plastique [Tableau II].

Ces essais de laboratoire sur des mélanges a solrésidus de bauxite ont mis er évidence certaines propriếtés intéressantes et bénéfiques du matériau: l'indice de portance CBR augmentait sensiblement, de 12 à 34 , pour un sol semi-grenu (A-2-7) à la présence de résidus de bauxite et la résistance en compression sirmple passait de 0,7 à $5,5 \mathrm{~kg} / \mathrm{cm}^{2}$ pour un matériau grenu (A-1) de faible plasticité (Mouratidis et Tsohos, 2002).

L'évaluation des résultats des essais, jugés positifs et prometteurs, a donné lieu, quelques années plus tard. à une deuxième étape de recherche en laboratoire (2001-2003) en vue de la construction expérimentale dun remblai routier.

tanuen II Propriétés physiques des résidus de baluxite. Physical projerties of bauxite residues.

Granalnmétrie Litrites d'Atterberg Masse spéciligue Passant $\mathrm{No} .200(0,074 m m) \quad W_{1}=35-39$ $=80-100 \%$

$1_{1}=0$ (N)

$\gamma_{4}=3,4-3,3 t / m^{3}$

TABleall Composition chimique des résidus de bauxite. Chemical composition of baxite residues.

\begin{tabular}{|c|c|c|c|c|c|}
\hline $\begin{array}{c}\text { Constituant } \\
\text { (\%) }\end{array}$ & $\begin{array}{l}\text { ALGi } \\
\text { Gréce }\end{array}$ & $\begin{array}{l}\text { Reymolds } \\
\text { Etats-Unis }\end{array}$ & $\begin{array}{c}\text { Gargano } \\
\text { Italie }\end{array}$ & $\begin{array}{c}\text { Pechiney } \\
\text { France }\end{array}$ & $\begin{array}{l}\text { Oradea } \\
\text { Roumanie }\end{array}$ \\
\hline $\mathrm{Al}_{2} \mathrm{O}_{3}$ & 15 & 15 & 12 & 15 & 21 \\
\hline $\mathrm{Fe}_{2} \mathrm{O}_{3}$ & 51 & 55 & 46 & 30 & 40 \\
\hline $\mathrm{SiO}_{2}$ & 10 & 5 & 12 & 7 & 10 \\
\hline $\mathrm{TiO}_{2}$ & 5 & 5 & $\bar{l}$ & 12 & 5 \\
\hline $\mathrm{CaO}$ & 13 & 8 & I & - & 5 \\
\hline $\mathrm{Na}_{2} \mathrm{O}$ & 2 & 2 & 7 & - & 9 \\
\hline Divers & 4 & 10 & - & - & 10 \\
\hline
\end{tabular}


Durant cette deuxieme étape de recherche, un grand nombre de sols naturels ont été examinés et évalués en laboratoire, notamment des sols non cohérents A-1, A-2 et un us sol-type n semi-fin A-4. Par ailleurs, des essais de laboratoire ont mis en évidence des effets favorables sur la déformabilité et la plasticité des sols cohérents $(\mathrm{A}-4$ et $\mathrm{A}-6)$.

Des mélanges de ces sols et de résidus de bauxite ont constitue la base de l'analyse en laboratoire et de l'applicabilité en construction routière de ce matériau secondaire.

Les amalyses en laboratoire ont conduit aux conclusions suivantes. En présence dé résidus de bauxite

- les matériaux gremus montrent un accroissement de leur cohésion et de leur résistance au cisállement:

- les sols fins deviennent moins plastiques et plus résistants.

Suite $\mathrm{ane}$ longue période de recherche en laboratoire sur des échantillons de mélanges usols-résidus de bauxite $n$, deux formules ont été finalement retenues : un mélange de sol A-1 et de résidus de bauxite en proportion $60 / 40$ (\%) à cause du volume important du sous-produit absorbé (formule A) et une formule (B) de résidus de bauxite, en état originel, nécessitant un taux de $4 \%$ de cendre volarte pour améliorer la maniabilité et réduire l'humidité. Ën plus, il a été démontré que la présence de cendre volante contribuait à la génél"ation. d'une cohésion (Mouratidis, 2004) ; ce phénomène est essentiel pour lemploi du sous-produit en construction de remblais routiers. Cette deuxieme formule pouvait comporter un certain risque, mais elle a été jugée susceptible de mettre en évidence toute l"ampleur des applications possibles du matériau. Les valeurs optimales du dosage en résidus de bauxite ont été choisies par rapport ta la valeur maximale de lá densité sẻche $\gamma_{a}$ (Fig. 1), obtenule au cours des essats en laboratoire. Par ầlleurs, il ếtait clair que les caractéristiques mécaniques ne poseraient pratiquement aucun problène à la stabilité de l'ouvrage en terre projeté.

Une troisieme formule [C] d'un sol représentatif A-4, en état pur, a été également retenue pour servir de base d'évaluation de l'expérience-pilote.

Des essais de compression simple sur un mélange d'un sol grenu (A-1) et d'un sol cohérent (A-4) ont mon w tré l'action de type " liant hydraulique n des résidus de bauxite (Fig. 2). Des valeurs importantes de résistance en compression simple ont été enregistrées lors des essais sur des éprouvettes de 7 et 14 jours (Tableau III)

Les résultats des essais CBR sur des échantillons constitués de mélanges réalisés selon les formules retenues sont donnés dans le tableau III. Des essais de cisaillement à la boite ont également été réalisés afin de fournir les données nécessajres aux calculs de stabilité du remblai.

Les valeurs des caractéristiques de cisaillement de tous les matériaux se sont révélées assez élevées et, par conséquent, aucun problème de stabilité ne semblait se poser a priori pour la construction du remblai.
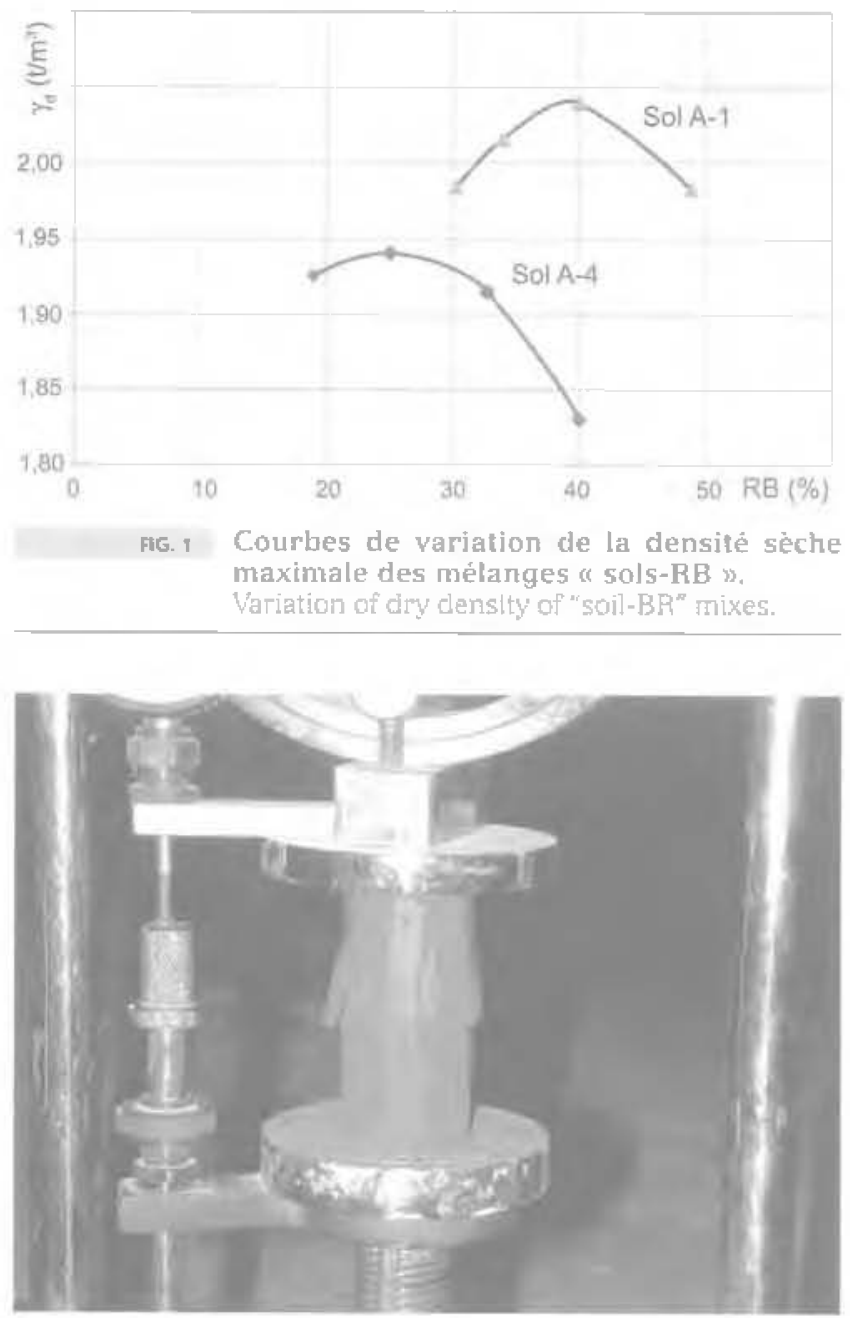

ॠG. I Essai de compression simple. Test of unconfined compression.

\section{3}

\section{Construction du remblai expérimental}

La construction du remblai routier expérimental s'est déroulée en avril et mai 2003, au sein du réseau routier local de Boétie. Le remblal a une hauteur de $2 \mathrm{~m}$ et ure largeur de plateforme de $8 \mathrm{~m}$ (Fig. 3). La longueur totale est de $100 \mathrm{~m}$ et comprend deux rampes d'accès de part et d'autre du remblai dans le sens de l'axe de la route locale.

Les résidus de bauxite ont étẻ récupérés dies lagunes de séchage au voisinage du site de construction et transportés sur le chantier de l'ouvrage expérimental (Fig. 4). Les sols naturels constitutifs du remblai ont été empruntés aux sites voisins du chantier.

Le remblai a été construil en trois sections distinctes: deux sections pour l'application des formules A et $B$ et une troisiene u section-type n constituée par un sol semi-fin du groupe A-4 (formule C).

L'équipement de construction á été assez modeste à cause de la taille limitée de l'ouvrage. Chargeuse à pneus et compacteur léger ont constitué l'essentiel du matériel de construction. Le remblai a été construit en couches de $40 \mathrm{~cm}$. 
TABLAaU II Effets de stabilisation sur mélanges " sol-résidus de bauxite ». Stablizing effects of "soil-beuxite residues" mixes.

\begin{tabular}{|c|c|c|c|c|c|c|c|}
\hline & \multirow{2}{*}{ Matérian/mélange } & \multicolumn{2}{|c|}{ Proctor Modifié } & \multirow{2}{*}{ CBA!* } & \multicolumn{3}{|c|}{ Compression simple $\left(\mathrm{kg} / \mathrm{cm}^{\mathrm{C}}\right)$} \\
\hline & & $\gamma_{n}\left[t / m m^{1}\right]$ & $W_{1 \text { int }}[\%]$ & & $\mathrm{q}_{\mu}\left[{ }^{*}\right]$ & 7 jours & 14 jours \\
\hline 1 & Résidus de Bauxite [RB] & 1,9 & 24 & 16 & - & - & - \\
\hline \multirow{2}{*}{2} & $A_{-1}=1$ & 2.2 & 6,7 & 39 & 0,7 & - & - \\
\hline & $\begin{array}{l}\mathrm{A}-1+\mathrm{RB} \\
(90 / 10)\end{array}$ & 2.2 & 7.2 & - & 5.5 & 8.2 & 21 \\
\hline 3 & $A-1+R B(150 / 40)$ & 2.1 & 16 & 34 & 5,5 & - & - \\
\hline \multirow{2}{*}{4} & $A-2-6$ & 1,6 & 15 & 12 & 1,85 & - & - \\
\hline & $\begin{array}{l}A-2-4+\mathrm{AB} \\
(50 / 50)\end{array}$ & 1,75 & 22 & 34 & - & 2,75 & - \\
\hline \multirow{2}{*}{5} & $A_{-}-2-7$ & 1,6 & 15 & 12 & - & - & - \\
\hline & $\begin{array}{l}\mathrm{A}-2-7+\mathrm{AB} \\
(50 / 50)\end{array}$ & 1,75 & 22 & 34 & & & \\
\hline \multirow{2}{*}{ G } & A. 4 & 2,01 & 9,2 & 22 & 5,6 & - & - \\
\hline & $\begin{array}{l}A-4+R B \\
(75 / 25)\end{array}$ & 196 & 15 & 32 & & 12,2 & 18,3 \\
\hline 7 & $\mathrm{~PB}+\mathrm{CV}(4 \%)$ & & & 16 & 3,8 & - & - \\
\hline
\end{tabular}

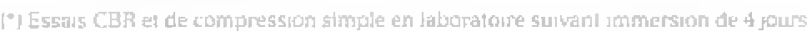

TARLEAU IV Caractérïstiques de cisaillement à la boîte. Shear strength characteristics.

\begin{tabular}{|c|c|c|c|}
\hline & Matériau & $\begin{array}{l}\text { Cubeficion } \\
\mathrm{c}^{\prime}\left(\mathrm{kg} / \mathrm{cm}^{2}\right)\end{array}$ & $\begin{array}{c}\text { Angle de frottement } \\
\varphi^{\prime} \text { (degrés) }\end{array}$ \\
\hline 1. & $A-1+A B(60 / 40)$ & 0,72 & 38 \\
\hline 2. & $\mathrm{~PB}+\mathrm{CV}(4 \%)$ & 0.43 & 41 \\
\hline 3. & $A-4$ & 0.56 & 32 \\
\hline
\end{tabular}

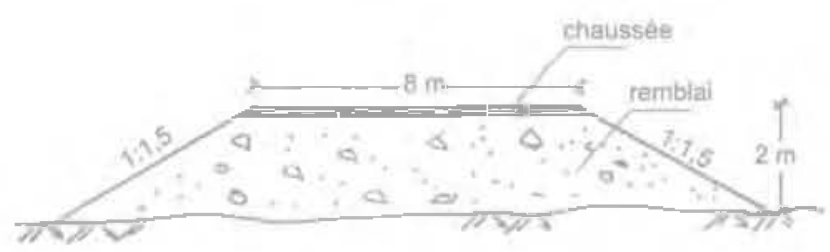

FG. 3 Coupe transversale du remblai.

Transverse section of the enbankment.

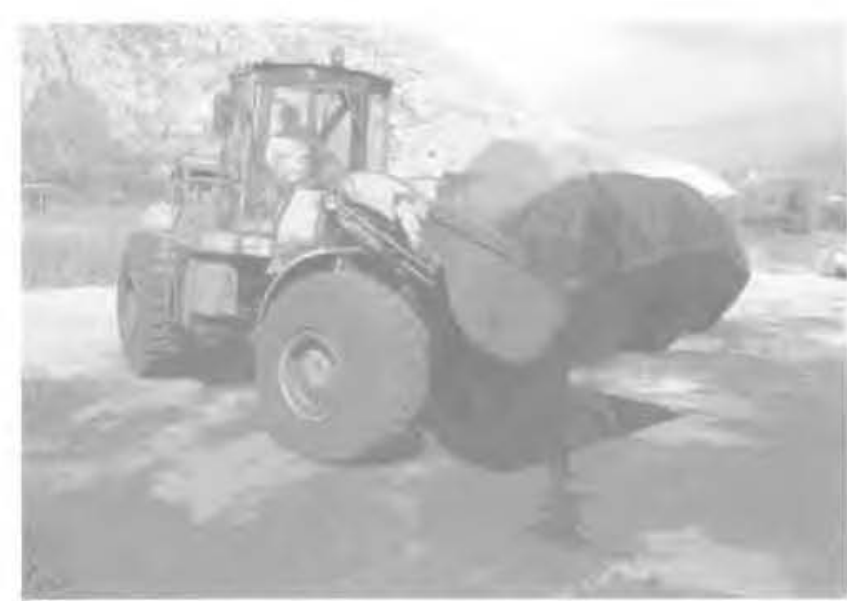

10. Apport de matériau. Transfert of material.
Les terrassements ont été effectués en période relativement sèche de manière conventionnelle et sous contrồle continu du taux d'humidité du matériau. L'humidité des résidus de bauxite variait de 16 à $22 \%$.

L'engin de compactage, un compacteur à cylindres jisses de 3 tonnes, a produit un compactage d'intensité moyenne, le degré de compactage variant de $88 \%$ à $93 \%$ de l'optimum Proctor modifié (Fig. 5).

Pendant la realisation de l'ouvrage en terre, la maniabilité des matériaux ainsj que leur mise en place en couches horizontales n'onł présenté aucun problème.

La totalifé des travaux a été réalisée en quelques Semaines. Les matériaux utilisés et, surtout, jes mélanges constitués de résidus de bauxite n'ont pas présenté de fissures de retrait juste après l'exécution des travaux. Aucune mesure spéciale d'entretien à court terme n'áété prise.

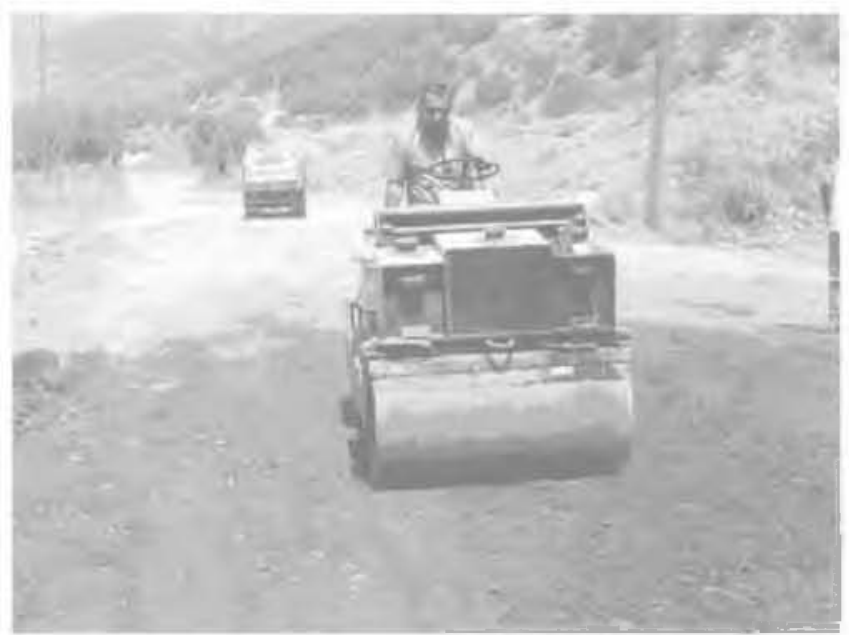

ma. Compactage des couches de remblai. Compaction of ambankment layers. 
La finalisation de l'ouvage comportait aussi la mise en place d'une couche de terre végétale sur les pentes du remblai afin de protéger l'ouviage contre l'érosion.

Comme le site expérimental choisi s'inscrit dans une installation industrielle, la route subit une circulation importante de poids lourds, estimée entre 30 et 50 poids lourds par sens at par jour. Il a donc été jugé nécessaire de recouvir la plate-forme d'une couche de grave non traitée de $20 \mathrm{~cm}$, constituée de gravillons de bauxite, pour recevoir et répartir les charges inportantes dues aux camions. L'ouvage a ensuite été ouvert à la circulation.

\section{4}

\section{Contrôle de qualité}

Des essais en place et en laboratoire ont été réalisés tout au long de la phase de construction. En dehors des contrôles d'humidité continus et des essais de compactage périodiques, une série d'essais de résistance mécanique en placé a êté effectuée. Léquipement utilisé était assez simple et se composait d'un pénétromètre CBR et d'un pénétromètre autoforeur. Les résultats des mesures, en valeurs moyennes, sont donnés dans le tableau $\mathrm{V}$.

Le comportement des matériaux en place a été jugé satisfaisant bien que les valeurs des mesures solent inférieures à celles du laboratoire. La résistance mécanique des mélanges comportant des résidus de bauxite est très élevée et indique des matêriaux adéquats à la construction de remblais routiers. Par conséquent, le comportement mécanique ne doit pas constituer un objet de préoccupation pour ce genre de construction. En effet, les points critiques de cette procédure ont óté :

- la maniabilité des matériaux.

- le contrôle de l'bumidité du sous-produt ;

- le compontement à long-terme et le risque d'ẻrosion.

Des contrôles systématiques et des mesures efficaces à cet égard sont nécessaires afin d'assurer une performance de haut niveau de l'ouvrage er terre.

\section{5}

\section{Comportement sous charges de trafic}

Lá chaussée a été mise sous circulation après Sa construction. Les véhicules lourds sur la voute de service produisaient un trafic journalier de $30-50$ poids lourds par sens et ont exercé des charges importantes sur la chaussée et la plateforme du remblai.

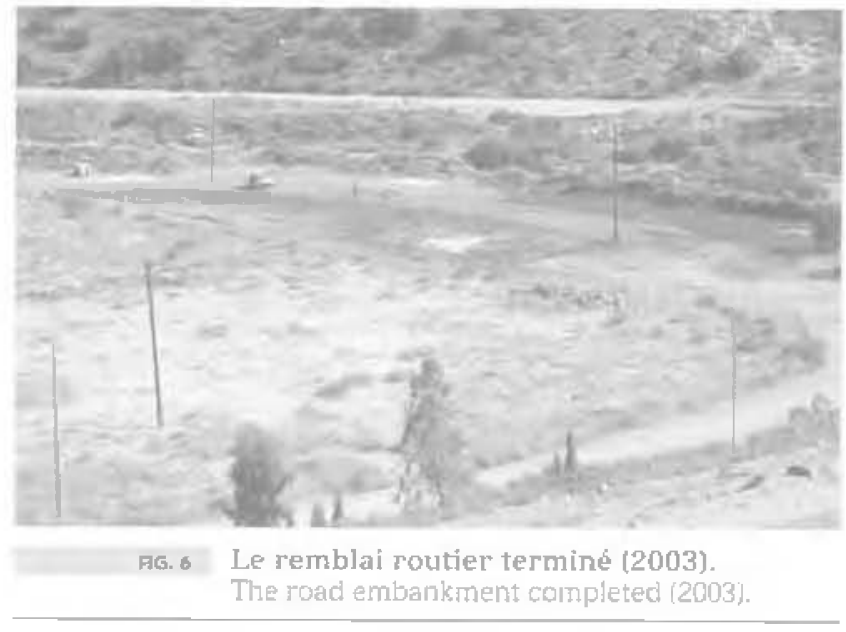

Un an aprés la mise en circulation. la structure du remblai me portait aucun signe visible de défaut ou de détérionation. Des mesures de tassement ont été effectuées sur les bords de la chaussée. Les valeurs du tassement total, dú principalement à la déformabilité du remblai, variaient de 1 à $2 \mathrm{~mm}$, ce qui démontre la durabilité de l'ouvage construit.

De même, cing ans après la mise sous trafic du remblai routier, Jes tassements mesurés en plateforme variaient de 4 à $11 \mathrm{~mm}$, ceci dû principalement à l'érosion superficielle subie dans le temps (Kehagia, 2008). Le revêtement des pentes en terre végétale s'est aussi bien comporté et aucune trace de dégradation par érosion n’a pu être ciscernée.

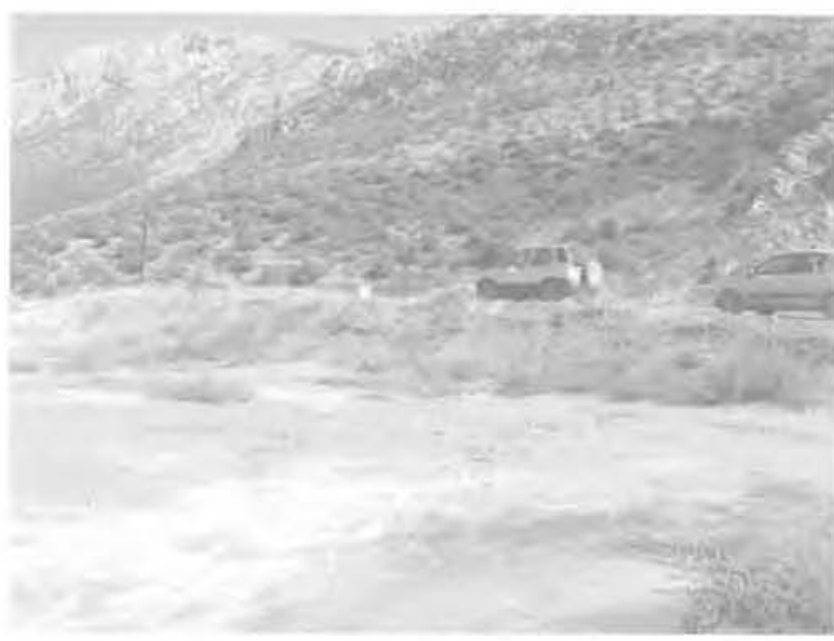

mt. 7 Le remblai routier cinq ans après (2008) The road embankment five years alter construction (2008).

TABLEAU v Mesures en place des caractéristiques mécaniques. Or site strencth characteristics.

\begin{tabular}{|c|c|c|c|c|c|}
\hline & \multirow{2}{*}{ Matนื้riaนt } & \multicolumn{2}{|c|}{ Indice CBR } & \multicolumn{2}{|c|}{ Compression simple $(\mathrm{kg} / \mathrm{cm}$ ' $)$} \\
\hline & & Laboratoire & En plece & Laboratoire & Eni place \\
\hline 1 & Sol A-1 + RB $160 / 401$ & 34 & $>15$ & 5,5 & 4,5 \\
\hline 2 & RB + Cendre volante & 16 & 13 & 1,8 & 3.5 \\
\hline 3 & Sol A4 & 27 & 315 & 5,6 & 5.0 \\
\hline
\end{tabular}


Globalement, la structure du remblai est restée intacte sous l'effet des charges de trafic et des internpéries. Cette performance de l'ouvrage, suite à une procédure de construction simple et facile à mettre en ouvre, remet en question la classification des résidus de bauxite placés en classe III des sous-produits industriels par rapport à leur aptitude d'application. en technique routière (OCDE, 1977). Cette classe comprend des matériaux secondaires de qualité médiocre qui peuvent être utilisés, seulement, dans des cas exceptionnels. L'expérience prẻsentée ici démontre, su contraire, qu'il suffit d"un traitement de séchage pour que le matériau puisse être appliqué en technique routière de façon simple et efficace. Les résíclus de bauxite devraient donc être considérés comme un sous-produit de classe 1 .

\section{6}

\section{Conclusion}

La construction d'un remblai routier expérimental en résidus de bauxite a dêmontré de façon claire la possibilité d'utilisel" ce sous-produit industriel en technique routière. L'utilisation de ce matériau a secondaire o peut donc s'avérer non seulement bénéfique vis-ă-vis de l'environmement, máis Égälement efficace en terme de perfomance technique à long terme. En effet, la structure du remblai n'a présenté aucun signe de détérioration cinq ans aprês sa construction et sa mise sous trafic.

La perspective d'une application systématique de ce matériau se heurte toutefois au cout du transport. Le sous-produit peut constituer un natériau de construc. tion dans la région de Boétie et, en général, au voisinage des installations de production industrielles. Pour une utilisation plus gênérale, il faudra trouver un moyen de subventionner le coût de transport selon une stratégie dirigée par les autorités locales, régionales et probablement aussi par l'industrie de production.

\section{Bibliographie}

Jitsangiam P., Nikraz. H., Jamieson E., Kitanovich R., Sirignin K. - Sustainable use of a Bauxite Residue (Red Sand] as Highway Embankment Materials. New Zealand Socjety for Sustainable Engineering and Science Conference. Auckland, 2008.

Kehagia F. - An iлnovative geotechnica! application of Bauxire Residue. EJGE Journal, vol. 13, 2008.

LRPC d'Aix-en-Provence - Utilisation des résidus inertes en technigue routière. Rapport technique, 1997, $90 \mathrm{p}$
Mouratidis A., Tsohos G. - Investigation of red mud properties for use in road construction. Proceedirgs $4^{\text {th }}$ finternational Gungress on Erironmental Geotechrics, Rio de Janeiro, 2002, p. 493-406.

Mouratidis A. - Construction expêrrinentale d"un remblali routier aux résidus de bauxite Compleg rendus de la confé. rence sur l'emploi des sous-produits industriels en constmation des rolutes. Thessalonique, 2004, p. 73-81.
Newson T., Dyer T.. Aden C., Sharg S. Effect of structure on the Geotechnical Properties of Bauxite Residue. Journal of Geotechnical and Geoenvironnental Enginesring, $\pi^{\circ} 132,2006$, p. 143-150.

OCDE - Utilisation des déchets en sous. prodults en technique routière. Rapport d'un groupe d'expert5. Paris. 1977. $174 \mathrm{p}$. 